\section{Visualized protein polymorphisms in leaf sheaths and roots of rice assessed by 2-DE analysis}

\author{
Fangyu Chen ${ }^{1}$, Liangrong Jiang ${ }^{2}$, Rongyu Huang ${ }^{2}$, Xiaolong Chen ${ }^{2}$, \\ Zhiming Chen ${ }^{1}$, Houcong Wang ${ }^{2}$, Yumin Huang ${ }^{2}$ and Jingsheng \\ Zheng $^{2^{*}}$
}

\begin{abstract}
To identify potential protein markers associated with tillering in rice, a d10 allelic mutant (JHCA) with a high-tillering dwarf phenotype and a wild-type cultivar (GLA4) with normal numbers of tillers were used to produce a hybrid $\left(F_{1}\right)$, and 2-DE (two-dimensional electrophoresis) analysis was conducted in seedling leaf sheaths and roots of the rice hybrid and its parents. A total of 61 protein spots representing qualitative or quantitative polymorphisms were detected. Among them, 38 spots were successfully identified as 23 different proteins by MALDI-TOF/MS. The identified proteins were further divided into P/As (presence/absence variations), PSs (position shift variations) and qVs (quantitative variations). Most of the identified polymorphic proteins exhibited tissue-specific expression. Two spot families, identified as a putative vitamin B12-independent methionine synthase and a putative phosphoglycerate mutase were found. The profiles of expression abundance of three iso-spots in each spot family showed a reciprocal relationship between the two parents.
\end{abstract}

Keywords: Protein polymorphism, rice, leaf sheath, root, proteomics.

\section{INTRODUCTION}

In the postgenomic era, a proteomic approach based on two-dimensional electrophoresis (2-DE) and mass spectrometry (MS) is a large-scale, highthroughput analysis tool for functional genomics. This approach provides very useful information from gene expression to cell metabolism and genetic linkages, and has consequently become a link among genomics, genetics, and physiology. Unlike the widely used DNA molecular markers in plant genetic research and breeding (Garrido-Cardenas et al. 2017, Kumar et al. 2017), proteomic polymorphisms based on 2-DE are real-time expression markers; therefore, the protein markers are beneficial for defining the relationships of molecular genetic markers with their physiological function. Furthermore, the method can generate novel insight and be useful for crop improvement. The potential of crop proteomics for the development of novel markers for plant breeding has not yet been fully realized. To better understand the molecular mechanisms of heterosis, proteomics has been applied as a powerful research tool to compare the patterns of protein accumulation in hybrids and their inbred lines, and many polymorphic protein markers have been reported in diverse organs of different plants, including roots and leaves in wheat (Song et al. 2007,
Crop Breeding and Applied Biotechnology

19: 110-117, 2019

Brazilian Society of Plant Breeding. Printed in Brazil http://dx.doi.org/10.1590/198470332019v19n1a15

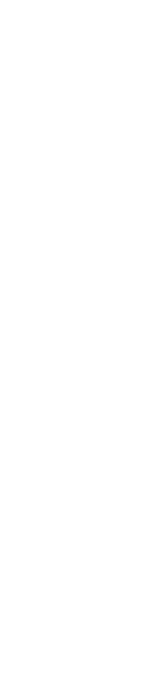


Song et al. 2009), roots and embryos in maize (Marcon et al. 2010), and leaves, endosperms and embryos in rice (Xie et al. 2006, Wang et al. 2008, Zhang et al. 2012). Proteomic analysis of a segregant population revealed candidate proteins linked to mealiness in peach (Almeida et al. 2016).

Rice is one of the most important crops in the world and is also an important model plant for monocots. In rice, the number of tillers is an important agronomic trait for grain production. Consequently, precisely regulated tiller numbers in rice are beneficial for increasing the yield. Many breeders and scientists focus on rice tillering control. Strigolactones (SLs) that are transported from the root to inhibit shoot branching have been identified as a new class of plant hormones in higher plants and were confirmed as endogenous regulators of plant growth and development (Waters et al. 2017). Genes involved in SL biosynthesis and perception have been identified following investigations on increased-branching mutants in Arabidopsis, pea, petunia and rice (Ruyter-Spira et al. 2013). In rice, decreased strigolactone levels in recessive $d 10$ mutants are attributable to a direct blockage of the biosynthesis pathway and lead to a high-tillering dwarf phenotype (Arite et al. 2007). The leaf sheath is the tissue responsible for elongation and important transmission regulation in rice plants (Shen et al. 2002). Previous studies have revealed that SLs are mobile signals produced in roots and move acropetally in shoots to inhibit lateral bud outgrowth (Waters et al. 2017). Accordingly, many critical metabolic and regulatory activities that control plant architecture might take place in the leaf sheaths and roots in rice. In this paper, a rice $d 10$ allelic mutant (JHCA), a wild-type cultivar (GLA4) and their hybrid progeny $\left(\mathrm{F}_{1}\right)$ were used for investigating polymorphic proteins in the leaf sheaths and roots by a proteomic approach and to build a foundation for further screening of potential biomarkers for tillering regulation in rice.

\section{MATERIAL AND METHODS}

\section{Plant material and sampling}

A high-tillering dwarf $d 10$ indica rice mutant (JHCA), semidwarf indica rice cultivar with normal tillers (wild-type, GLA4), and their hybrid progeny $\left(F_{1}\right)$ were used in the current research. JHCA was obtained through mutation breeding by irradiation with gamma $(\gamma)$ rays. In earlier studies, JHCA was demonstrated to carry two recessive dwarf-related genes, namely, $d 10$ and $s d 1$. DNA sequencing showed that the recessive $d 10$ locus contains a 39-bp deletion at the second exon of D10 (LOC_Os01g0746400), which led to a frame-shift mutation (Chen et al. 2013). The tiller number of each JHCA plant is greater than 120 , and the plant height is approximately $45 \mathrm{~cm}$ (Figure 1C). The wild-type cultivar GLA4 carries the recessive semidwarf gene sd1 only. The tillering characteristics and plant height of $F_{1}$ are similar to those of GLA4 (Figure 1A-C).

Pregerminated rice seeds were sown in the field, and the seedlings were transplanted with a space of approximately $20 \times 20 \mathrm{~cm}$ per plant. The roots and leaf sheath of the third leaf from plants in the plot were harvested at the active tillering stage ( 30 days after transplanting), frozen in liquid nitrogen and stored at -80 ${ }^{\circ} \mathrm{C}$ prior to protein extraction.
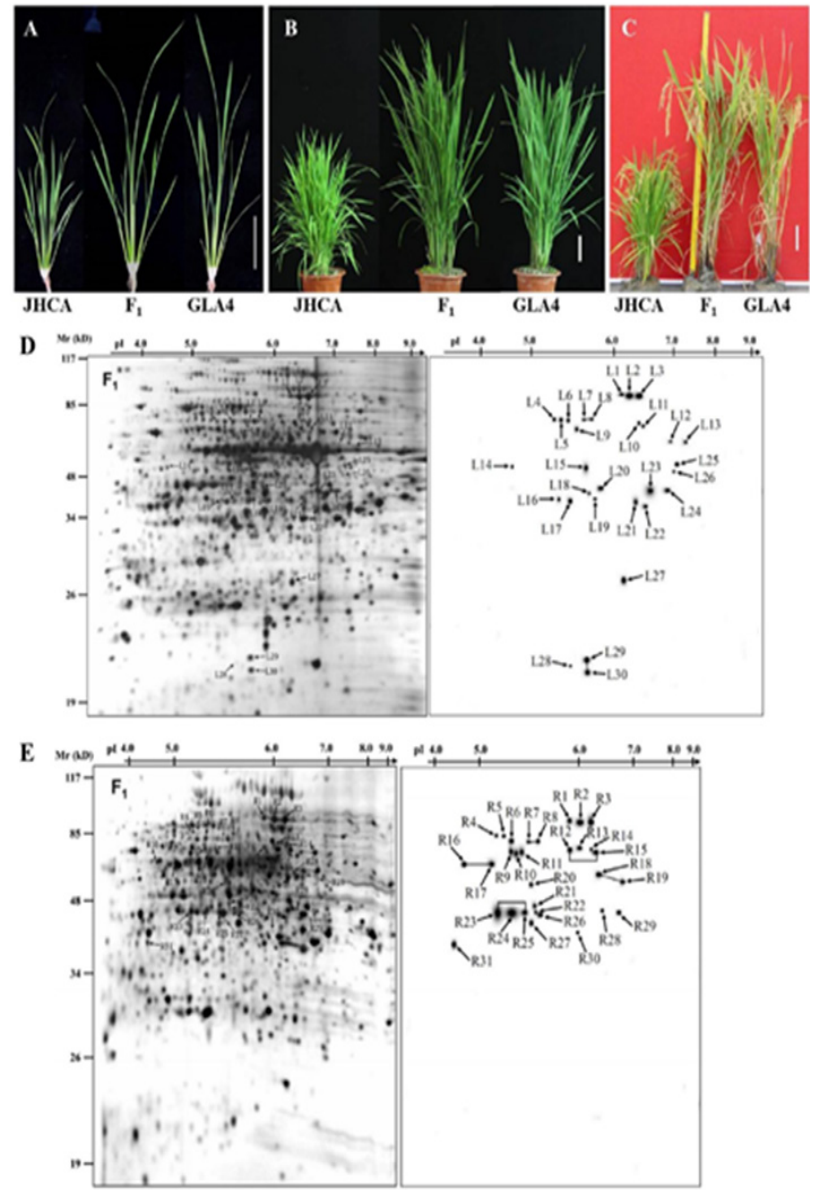

Figure 1. Phenotypes of JHCA, GLA4 and the hybrid $\mathrm{F}_{1}$ at different developmental stages and 2-DE maps of leaf sheaths and roots from the hybrid $F_{1}$. A, B and C show the phenotypes of JHCA, GLA4 and the hybrid $F_{1}$ in the early tillering stage, maximum tillering stage and maturity stage, respectively. Bar $=10 \mathrm{~cm}$. D and E show the 2-DE maps and the distribution of protein variants in leaf sheaths and roots of the hybrid $\mathrm{F}_{1}$, respectively. The dashed lines show the diallelic loci of position shift (PS). 


\section{Protein extraction, two-dimensional electrophoresis and image acquisition}

Total proteins were extracted in extraction buffer and precipitated by trichloroacetic acid. The pellets were dissolved in lysis buffer containing $7 \mathrm{M}$ urea, $2 \mathrm{M}$ thiourea, 4\% (w/v) CHAPS, $65 \mathrm{mM}$ DTT, and Bio-Lyte pH 3-10 (Bio-Rad). The protein content of the soluble fraction was determined using a BCA Protein Assay (Pierce). A total of $150 \mu \mathrm{g}$ of protein was loaded onto IPG strips (pH 3-10NL, $17 \mathrm{~cm}$, Bio-Rad). Isoelectric focusing (IEF) was performed using a Protean IEF Cell (Bio-Rad). Then, IPG strips were equilibrated in $50 \mathrm{mM}$ Tris- $\mathrm{HCl}(\mathrm{pH} 8.8), 6 \mathrm{M}$ urea, $30 \%$ glycerol, $2 \%$ SDS, and bromophenol blue by shaking two times, the first with $2 \%$ DTT (15 min) and the second with $2.5 \%$ iodoacetamide (15 $\mathrm{min})$. The second-dimension electrophoresis was carried out with $12 \%$ SDS-PAGE at a constant current of $25 \mathrm{~mA}$ for approximately $5 \mathrm{~h}$. Proteins were visualized by silver staining, scanned, and analyzed for differentially expressed spots using PDQuest 7.1 software (Bio-Rad). The spots present in all three biological replicates showing statistically significant, qualitative or quantitative differences were visualized after comparisons. Data analysis was performed with Excel software (Microsoft). The error bars shown in figures represent the standard deviation values.

\section{Mass spectrometry analysis and database search}

Selected protein spots detected on the 2-DE maps were excised from the gels and digested with sequencing-grade trypsin (Promega). Mass spectrometry analysis and database searches of the peptides were carried out as described by Chen et al. (2014).

\section{RESULTS AND DISCUSSION}

\section{Detection of protein polymorphisms in leaf sheaths and roots of rice}

Highly reproducible 2-DE patterns with high resolution were obtained from leaf sheaths and roots of JHCA, GLA4 and their hybrid $F_{1}$. Over 1,200 and 1,000 protein spots were reproducibly detected in 2-DE patterns for leaf sheaths and roots, respectively. Typical 2-DE separation of proteins from hybrid $F_{1}$ samples is shown in Figure 1 . The differential protein spots among JHCA, GLA4 and hybrid $F_{1}$ samples of leaf sheaths and roots are shown in Figure 2A-B. Following a detailed analysis with PDQuest 7.1 software, a total of 30 and 31 spots were detected from leaf sheaths and roots, respectively, with differential expression levels in 2-DE maps among the two parents and hybrid $\mathrm{F}_{1}$. Furthermore, all these differentially expressed proteins were found in the hybrid $F_{1}$. They were assigned numbers with $L$ (L1 to L30) for leaf sheath and R (R1 to R31) for roots in 2-DE maps of the hybrid $F_{1}$. The distribution of protein variants in the 2-DE maps is also shown in Figure 1. These polymorphic proteins can be divided into qualitative and quantitative variations (Table 1 and Figure 2). As shown in Figure 2A, spots L10 and L11 both showed qualitative variation patterns with presence/ absence variations (P/As). They both showed 'absence' in JHCA but 'presence' in GLA4 and showed 'presence' in the hybrid $F_{1}$. Therefore, ' $P$ ' is dominant over ' $A$ ', and $P / A s$ are monogenic and dominant molecular markers. Spots $L 7-L 8$ in Figure $2 \mathrm{C}$ and spots L29-L30 in Figure 2D also exhibited qualitative variations with P/A character. As shown in Figure 2E, spot L27 showed quantitative variation (qV) in the relative abundance. The relative abundance of spot L27 was 'high' in JHCA but 'low' in GLA4 and 'high' in the hybrid $F_{1}$. Therefore, a high amount was dominant over a low amount, and qVs were also monogenic and dominant molecular markers.

Among the 44 qualitative variation spots, which showed as presence/absence variations (P/As), 21 were uniquely expressed in JHCA (eight in leaf sheaths and 13 in roots), and 23 were uniquely expressed in GLA4 (13 in leaf sheaths and ten in roots) (Table 1). Among the 17 quantitative variation (qV) spots, ten had a higher abundance in JHCA (six in leaf

Table 1. Polymorphic protein spots in leaf sheaths and roots from the two parents

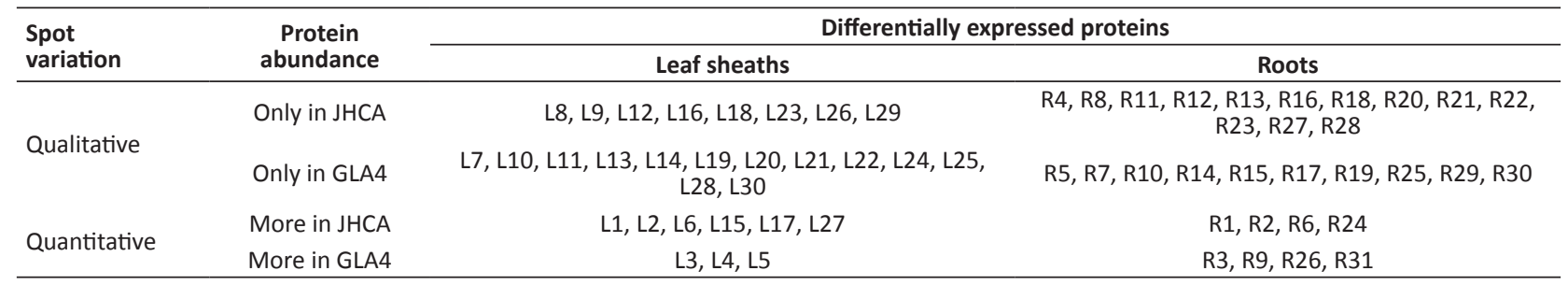


sheaths and four in roots), while seven were higher in GLA4 (three in leaf sheaths and four in roots). In the hybrid $\mathrm{F}_{1}$, all the P/As and qVs were 'presence' (P) and 'higher', respectively. All the polymorphic protein spots displayed a mirrored relationship between the two parents and $\mathrm{F}_{1}$, showing the genetic linkage among them.

\section{Mass spectrometric identification of polymorphic proteins and analysis of variations}

Identification of these polymorphic protein spots was carried out by means of MALDI-TOF/MS and online searching. Among the 61 total protein spots, 38 spots ( 20 in leaf sheaths and 18 in roots) were successfully identified as 23 different proteins (Table 2). A total of 14 different proteins in leaf sheaths and 13 prteins in roots were identified, and four of these proteins were common to both groups.
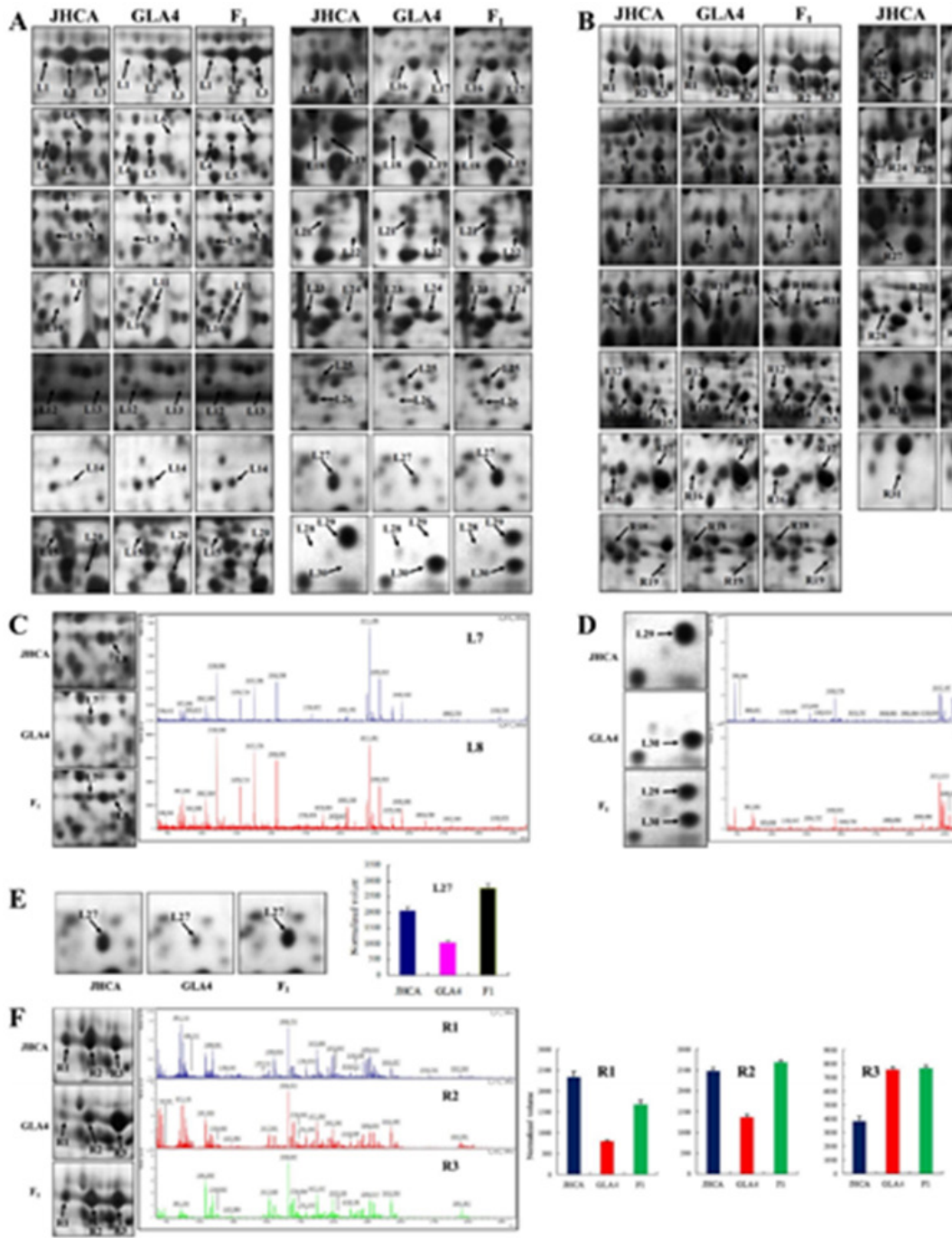
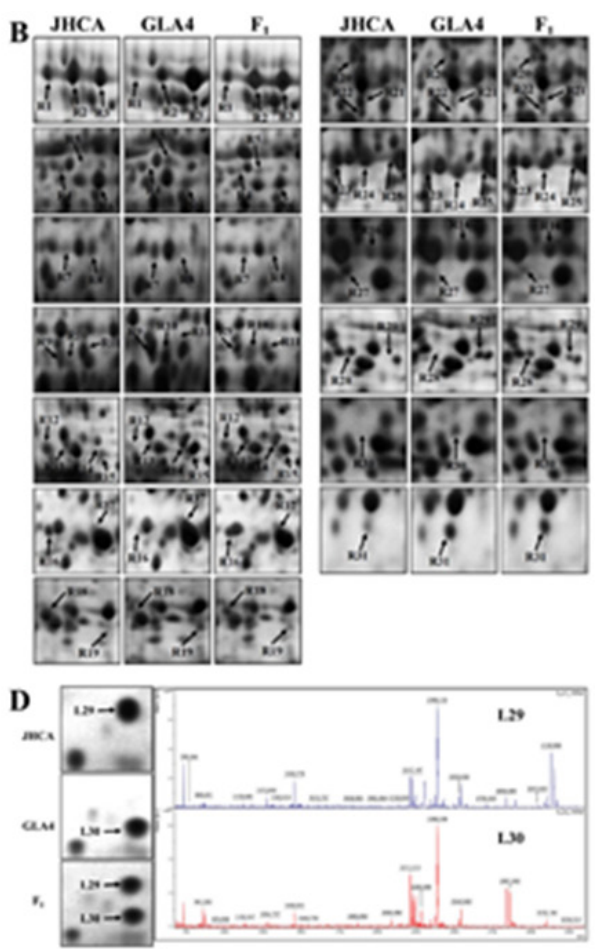

Figure 2. Differential expression of proteins among JHCA, GLA4 and the hybrid $\mathrm{F}_{1}$ in leaf sheaths and roots. A and B show the differential spots in leaf sheaths and roots, respectively. Arrows indicate the positions of protein spots showing differential expression. $C$ and $D$ show the identification of position shift variations (PSs) in the horizontal and vertical dimensions with peptide mass fingerprinting, respectively. The two spots from JHCA and GLA4 with identical migration upon 2-DE display similar mass signals, indicating that they are from the same protein shown with 2-DE. E: Quantitative variations (qVs). F: Quantitative differences in the three iso-spots of a spot family. The three iso-spots have similar mass signals and are shown as qVs. 
F Chen et al.

Table 2. Identification of polymorphic proteins by peptide mass fingerprinting ${ }^{a}$

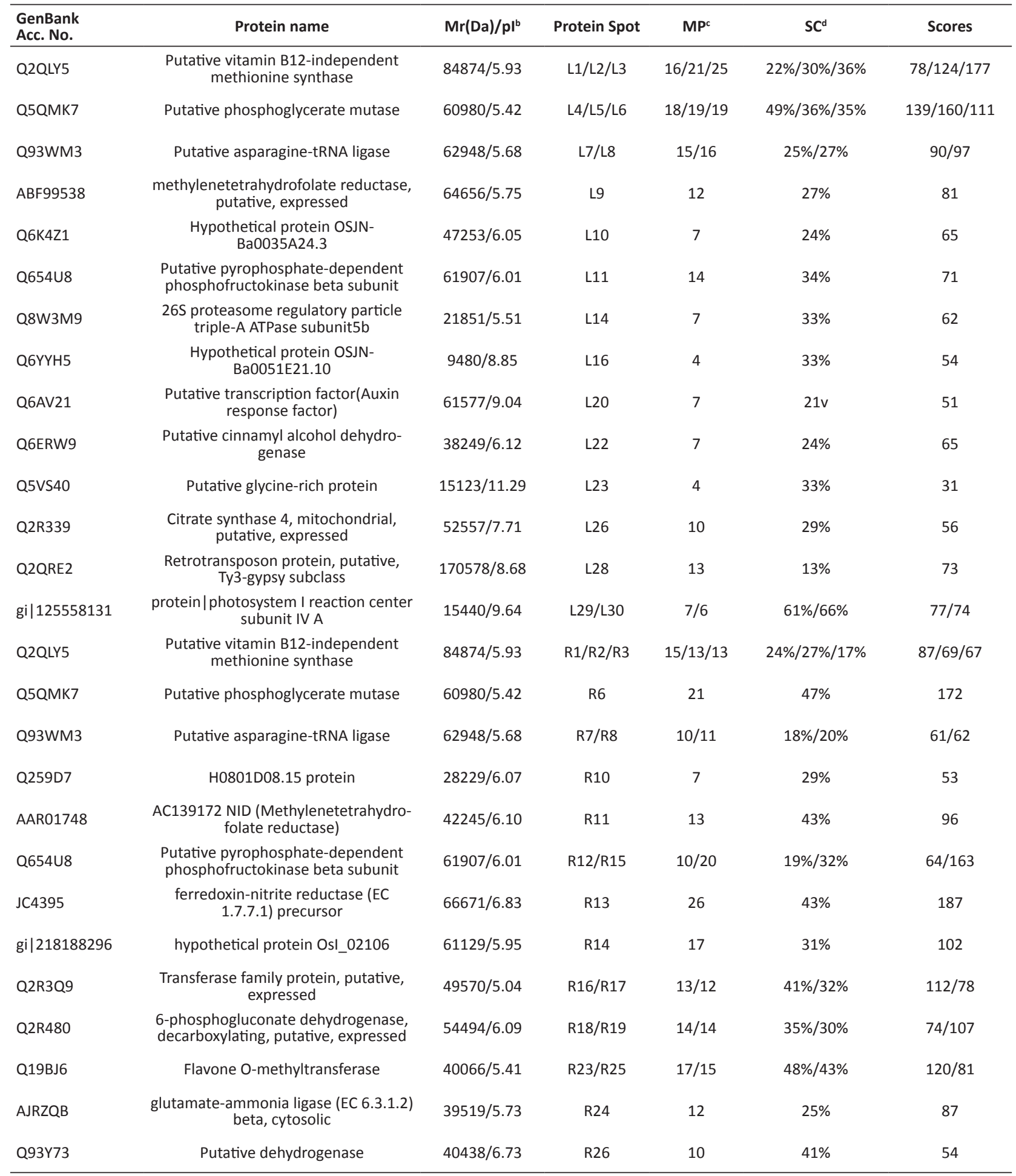

a Differentially expressed protein spots were excised from 2-DE gels and subjected to peptide mass fingerprinting with MALDI-TOF/MS; ${ }^{b} \mathrm{Mr}$, theoretical relative molecular mass; pl, theoretical isoelectric point; ${ }^{\mathrm{c}} \mathrm{MP}$, matched peptides; ${ }^{\mathrm{d}} \mathrm{SC}$, sequence coverage. 
As illustrated in Figure 2C, a pair of P/A-type spots (L7-L8), with identical migration in the 2-DE horizontal dimension displayed similar peptide mass fingerprints (PMFs), indicating that they were from the same protein and encoded by codominant alleles. Therefore, spots L7 and L8 were identified as a pair of position shift variations (PSs). Figure 2D shows another pair of PSs composed of spots L29 and L30 in the vertical dimension. A total of 14 P/A-type spots were further confirmed as seven pairs of PSs (Table 1 and Table 2), including L7-L8, L29-L30, R7-R8, R12-R15, R16-R17, R18-R19 and R23-R25, which were all diallelic. Most of the PSs differed in their isoelectric points in the horizontal direction but had similar molecular weights, aspects, and quantities except L29-L30, which showed a vertical shift. L29-L30 had similar isoelectric points, aspects and quantities but differed slightly in their molecular weights.

In the 2-DE patterns, spots exhibiting qualitative variations (P/As and PSs) were shown to be under monogenic control. $\mathrm{P} /$ As present a monogenic and dominant inheritance pattern. Two spots in a pair of PSs further presented a monogenic and codominant inheritance pattern. Most of the PSs correspond to allelic differences in the primary structure of a protein due to mutations in the structural genes, while the vertical shift of PS is a special situation. A previous report indicated that the vertical shift did not result from molecular mass alterations but rather from changes in protein conformation or the shape of the SDS-protein complex (Klose et al. 2002).

$\mathrm{P} / \mathrm{As}$ are ambiguous because they may correspond either to PSs in which a member of the pair is not detected or to a quantitative variation as a consequence of the action of regulatory genetic elements in which the absent spot is below the level of detection by silver staining. Therefore, a P/A locus corresponds either to a structural gene of the protein or a regulatory gene controlling the expression level of the protein. Nevertheless, their genetic basis can be monogenic, and thus they can be used as dominant markers. Based on expression patterns in the 2-DE maps of the two parents and $F_{1}$, eight spots might also be proposed as four pairs of PSs, including L23-L24, L25-L26, R4-R5 and R28-R29, though neither or either of a pair of PSs was identified. Spots with no dominant identification with MALDI-TOF/MS were still divided into the P/A group. qVs are quantitative variations, which can be under the control of multiple loci (Damerval 1994). Previous studies showed that dominance was observed for most qVs, with high abundance dominant over low abundance in most cases, which is consistent with the observation that the relative abundances of proteins in the $F_{1}$ hybrids on average deviate towards the high parental values (de Vienne et al. 1988). This conclusion was also confirmed by our results. Twelve spots among the $17 \mathrm{qV}$-type variations were identified as four proteins. Two spot families, identified as a putative vitamin B12-independent methionine synthase (L1-L2-L3, R1-R2-R3) and a putative phosphoglycerate mutase (L4-L5-L6), were found. The three iso-spots in each spot family identified as the same protein (Table 2 and Figure $2 \mathrm{~F}$ ) were arranged contiguously in the horizontal dimension. Interestingly, the profiles of expression abundance of the three iso-spots all showed a reciprocal relationship between JHCA (mutant) and GLA4 (wild-type). For example, the R1-R2-R3 spot family showed 'high-high-low' (H-H-L) in JHCA but 'low-low-high' (L-L-H) in GLA4. In $\mathrm{F}_{1}$, three iso-spots among these two spot-families all showed high-high-high $(\mathrm{H}-\mathrm{H}-\mathrm{H})$. High abundance was clearly dominant over low abundance.

In summary, different allelic spot(s) of a protein or iso-spots of spot families were scored as one variation. Thus, a variation could be represented by a single P/A spot, a single qV spot, a pair of two allelic PS spots, or a qV spot-family with three iso-spots. A total of 24 variations in leaf sheaths (two PSs, $17 \mathrm{P} / \mathrm{As}$ and five qVs) and 24 variations in roots (five PSs, $13 \mathrm{P} /$ As and six qVs) were identified (Table 3).

\section{Polymorphism of tissue-specific and tissue-nonspecific peptides in leaf sheaths and roots}

It has been shown that organ-specific proteins exhibit greater genetic variation than organ-nonspecific proteins (Bahrman and Petit 1995). Our present results confirmed this conclusion. There were ten and nine tissue-specific

Table 3. Different types of protein polymorphisms from leaf sheaths and roots

\begin{tabular}{lcr}
\hline \multirow{2}{*}{ Types of variation } & \multicolumn{2}{c}{ Tissues } \\
\cline { 2 - 3 } & Leaf sheaths & Roots \\
\hline PSs & L7-L8, L29-L30 & R7-R8, R12-R15, R16-R17, R18-R19, R23-R25 \\
P/As & L9, L10, L11, L12, L13, L14, L16, L18, L19, L20, L21, L22, L23, & R4, R5, R10, R11, R13, R14, R20, R21, R22, R27, R28, R29, \\
qVs & L24, L25, L26, L28 & R30 \\
\hline
\end{tabular}


polymorphic peptides in leaf sheaths and roots, respectively. Nevertheless, only four common polymorphic peptides were tissue-nonspecific.

The four tissue-nonspecific peptides included a putative vitamin B12-independent methionine synthase (MetE, Q2QLY5), putative phosphoglycerate mutase (PGAM, Q5QMK7), putative asparagine-tRNA ligase (Q93WM3) and putative pyrophosphate-dependent phosphofructokinase beta subunit (Q654U8). The putative MetE was identified in a spotfamily in leaf sheaths and roots. The putative PGAM was shown to be in a spot-family only in leaf sheaths, while qV showed only one spot in roots. MetE is required for the biosynthesis of methionine and tetrahydrofolate in plants. The differential expression of MetE has been identified in 110 mutant seedlings of rice in response to SL treatment (Chen et al. 2014). PGAM is a monomeric enzyme. It catalyzes the interconversion of 3-phosphoglycerate and 2-phosphoglycerate and is independent of the cofactor 2,3-bis-phosphoglycerate in plants. This result suggested that there were more critical metabolism differences regulated by the tissue-nonspecific peptides. Further studies should be conducted to elucidate whether the reciprocal regulation of spot families between wild-type and mutant plants is related to rice tillering control. Putative asparagine-tRNA ligase showed PS (L7-L8, R7-R8) in both tissues. The putative pyrophosphate-dependent phosphofructokinase beta subunit showed PS (R12-R15) in the root and P/A (L11) in the leaf sheath.

In contrast, tissue-specific polymorphic proteins indicated the specific tissue function and variations between two parental cultivars. SLs were produced in roots and moved acropetally in shoots to inhibit branching (Waters et al. 2017). Therefore, the search for polymorphic proteins in these two tissues built a foundation for investigating potential protein markers that might be associated with $D 10$ or tillering traits in rice. For example, the leaf sheath-specific polymorphic peptide L20 spot, which was only observed in the wild-type cultivar GLA4, was identified as an auxin response factor (Q6AV21). A previous study showed that D10 promotes cytokinin biosynthesis by reducing auxin levels (Zhang et al. 2010). Many SL-related physiological effects are intimately related to auxin-dependent processes (Shinohara et al. 2013). The differential expression of the auxin response factor implied the differential regulation of auxin levels between $d 10$ mutant JHCA and wild type GLA4. L29-L30, a pair of PS variations in leaf sheaths with a vertical shift, was identified as photosystem I reaction center subunit IV A, which is a key member of photosystem I, which is involved in light harvesting during photosynthetic processes. This protein was also identified in a previous report on SL-regulated proteins revealed by iTRAQ-based quantitative proteomics in Arabidopsis (Li et al. 2014). In our study, the relationship between the special protein phenotype (vertical shift) of L29-L30 and tillering control in rice requires further study. L14, showing as a P/A variation in leaf sheaths, was identified as the $26 \mathrm{~S}$ proteasome regulatory particle triple-A ATPase subunit5b, an important component of the ubiquitin proteasome system. The SL signaling pathway is dependent on the ubiquitination of D53 and its subsequent degradation by the ubiquitin proteasome system (Waters et al. 2017). The root-specific polymorphic proteins ferredoxin-nitrite reductase precursor (JC4395, R13) and transferase family protein (Q2R3Q9, R16-R17) were shown as P/A and PS, respectively. In a previous report on SL-regulated proteins in Arabidopsis, two ferredoxin-like proteins (2Fe-2S ferredoxin-like protein and ferredoxin 1) and a transferase family protein were identified (Li et al. 2014). Another two root-specific polymorphic proteins, namely, AC139172 NID (AAR01748) and flavone O-methyltransferase (Q19BJ6) were identified as P/A (R11) and PS (R23-R25), respectively.

Our results suggested that many polymorphic genetic markers exist in leaf sheaths and roots in different rice genotypes. In addition, they laid a foundation for finding potential tillering-related protein markers in rice with population genetics and proteomics approaches. Moreover, the protein markers obtained from pedigree analysis based on a proteomic approach have panoramic characteristics and might have special significance for molecular marker-assisted selection (MAS) in crop breeding due to their visualized advantage.

\section{ACKNOWLEDGEMENTS}

This work was supported by the National Key R\&D Program of China (No. 2017YFD0100103). The authors are grateful to Dr. Changchuan Xie and Dr. Yaying Wu (Analysis and Testing Center, School of Life Sciences, Xiamen University) for their help in mass spectrometry analysis. 
Visualized protein polymorphisms in leaf sheaths and roots of rice assessed by 2-DE analysis

\section{REFERENCES}

Almeida AM, Urra C, Moraga C, Jego M, Flores A, Meisel L, González M, Infante R, Defilippi BG, Campos-Vargas R and Orellana A (2016) Proteomic analysis of a segregant population reveals candidate proteins linked to mealiness in peach. Journal of Proteomics 131: 71-81.

Arite $\mathrm{T}$, Iwata $\mathrm{H}$, Ohshima K, Maekawa M, Nakajima M, Kojima M, Sakakibara $\mathrm{H}$ and Kyozuka J (2007) DWARF10, an RMS1/MAX4/DAD1 ortholog, controls lateral bud outgrowth in rice. Plant Journal 51: 1019-1029.

Bahrman N and Petit RJ (1995) Genetic polymorphism in Maritime Pine (Pinus pinaster Ait) assessed by 2-dimensional gel electrophoresis of needle, bud, and pollen proteins. Journal of Molecular Evolution 41: 231-237.

Chen F, Jiang L, Zheng J, Huang R, Wang H, Hong Z and Huang Y (2013) Identification of a co-segregative protein associated with the tillering trait in rice (Oryza sativa L.). Plant Omics 6: 36-45.

Chen F, Jiang L, Zheng J, Huang R, Wang H, Hong Z and Huang Y (2014) Identification of differentially expressed proteins and phosphorylated proteins in rice seedlings in response to strigolactone treatment. PLoS ONE 9: e93947.

Damerval C (1994) Quantification of silver-stained proteins resolved by two-dimensional electrophoresis: Genetic variability as related to abundance and solubility in two maize lines. Electrophoresis 15: 1573-1579.

de Vienne D, Leonardi A and Damerval C (1988) Genetic aspects of variation of protein amounts in maize and pea. Electrophoresis 9: 742-750.

Garrido-Cardenas JA, Mesa-Valle C and Manzano-Agugliaro F (2017) Trends in plant research using molecular markers. Planta 247: 543-557.

Klose J, Nock C, Herrmann M, Stühler K, Marcus K, Blüggel M, Krause E, Schalkwyk LC, Rastan S, Brown SDM, Büssow K, Himmelbauer H and Lehrach H (2002) Genetic analysis of the mouse brain proteome. Nature Genetics 30: 385-393.

Kumar S, Saxena S and Gupta MG (2017) Marker-assisted screening of breeding populations of an apomictic grass Cenchrus ciliaris L. segregating for the mode of reproduction. Crop Breeding and Applied Biotechnology 17: 10-17.
Li Z, Czarnecki O, Chourey K, Yang J, Tuskan GA, Hurst GB, Pan C and Chen JG (2014) Strigolactone-regulated proteins revealed by iTRAQbased quantitative proteomics in Arabidopsis. Journal of Proteome Research 13: 1359-1372.

Marcon C, Schutzenmeister A, Schutz W, Madlung J, Piepho HP and Hochholdinger $F$ (2010) Nonadditive protein accumulation patterns in maize (Zea mays L.) hybrids during embryo development. Journal of Proteome Research 9: 6511-6522.

Ruyter-Spira C, Al-Babili S, van der Krol S and Bouwmeester H (2013) The biology of strigolactones. Trends in Plant Science 18: 72-83.

Shen S, Matsubae M, Takao T, Tanaka N and Komatsu S (2002) A proteomic analysis of leaf sheaths from rice. Journal of Biochemistry 132: 613-620.

Shinohara N, Taylor C and Leyser O (2013) Strigolactone can promote or inhibit shoot branching by triggering rapid depletion of the auxin efflux protein PIN1 from the plasma membrane. PLoS Biology 11: e1001474.

Song X, Ni Z, Yao Y, Xie C, Li Z, Wu H, Zhang Y and Sun Q (2007) Wheat root proteome and differentially expressed root proteins between hybrid and parents. Proteomics 7: 3538-3557.

Song X, Ni Z, Yao Y, Zhang Y and Sun Q (2009) Identification of differentially expressed proteins between hybrid and parents in wheat (Triticum aestivum L.) seedling leaves. Theoretical and Applied Genetics 118: 213-225.

Wang W, Meng B, Ge X, Song S, Yang Y, Yu X, Wang L, Hu S, Liu S and Yu J (2008) Proteomic profiling of rice embryos from a hybrid rice cultivar and its parental lines. Proteomics 8: 4808-4821.

Waters MT, Gutjahr C, Bennett T and Nelson DC (2017) Strigolactone signaling and evolution. Annual Review of Plant Biology 68: 291-322.

Xie Z, Wang J, Cao M, Zhao C, Zhao K, Shao J, Lei T, Xu N and Liu S (2006) Pedigree analysis of an elite rice hybrid using proteomic approach. Proteomics 6: 474-486.

Zhang C, Yin Y, Zhang A, Lu Q, Wen X, Zhu Z, Zhang L and Lu C (2012) Comparative proteomic study reveals dynamic proteome changes between superhybrid rice LYP9 and its parents at different developmental stages. Journal of Plant Physiology 169: 387-398.

Zhang S, Li G, Fang J, Chen W, Jiang H, Zou J, Liu X, Zhao X, Li X, Chu C, Xie $Q$, Jiang $X$ and Zhu $L$ (2010) The interactions among DWARF10, auxin and cytokinin underlie lateral bud outgrowth in rice. Journal of Integrative Plant Biology 52: 626-638. 